\title{
Anderson-Hines Open Pyeloplasty in the Treatment of Pyelo-Ureteral Junction Syndrome: Results from 36 Cases
}

\section{Anani Wencesl Severin Odzébé ${ }^{*}$, Caryne Mboutol-Mandavo ${ }^{2}$, Aristide Steve Ondziel Opara1, Lucie Irene Patricia Ondima ${ }^{2}$, Armel Melvin Ondongo Atipo ${ }^{1}$, Rolland Bertile Banga Mouss ${ }^{1}$, Prosper Alain Bouya ${ }^{1}$}

\author{
${ }^{1}$ Department of Urology and Andrology, University Hospital, Brazzaville, Congo \\ ${ }^{2}$ Department of Padiatric Surgery, Faculty of Health Science, University Marien Ngouabi, Brazzaville, Congo \\ Email: *odzebe_s@yahoo.fr
}

How to cite this paper: Odzébé, A.W.S., Mboutol-Mandavo, C., Opara, A.S.O., Ondima, L.I.P., Atipo, A.M.O., Mouss, R.B.B. and Bouya, P.A. (2019) Anderson-Hines Open Pyeloplasty in the Treatment of Pyelo-Ureteral Junction Syndrome: Results from 36 Cases. Open Journal of Urology, 9, 131-139. https://doi.org/10.4236/oju.2019.99016

Received: September 25, 2018

Accepted: September 16, 2019

Published: September 19, 2019

Copyright $\odot 2019$ by author(s) and Scientific Research Publishing Inc. This work is licensed under the Creative Commons Attribution International License (CC BY 4.0).

http://creativecommons.org/licenses/by/4.0/

(c) (i) Open Access

\begin{abstract}
Goal: To evaluate the results and complications of open pyeloplasty according to Anderson-Hynestechnic. Patients and Methods: We conducted a retrospective study from 2000 to 2014 . The study included 36 cases of opening the ureteropyelic junction operated pit syndrome according to Anderson-Hynes technique. Results: Lumbotomy was used in all patients. A pelvic pyelolithotomy for lithiasis was performed in two patients $(5.5 \%)$ and unwinding of a lower polar pedicle in 3 cases $(8.3 \%)$. The average duration of response was $119 \pm 15 \mathrm{~min}$. The average length of hospital stay was $11.2 \pm 3$ days. Patients were followed for a mean of 10 months. Thirty-five patients were asymptomatic and in one case lower back pain persisted. IVU to 6 months showed a permeable junction in $97.2 \%$ of cases. Seven patients (19.4\%) had short-term complications. Ureteropelvic stenosis was the only complication in the medium and long term in one case $(2.8 \%)$. The success rate of the Pyeloplasty was $97.2 \%$. Conclusion: The open pyeloplasty as Anderson-Hynes remains the treatment of choice in our context SJPU with great results. The indications tend to decrease in favor of laparoscopic pyeloplasty.
\end{abstract}

\section{Keywords}

Hydronephrosis, Pyeloplasty, Anderson Hynes, Open Surgery

\section{Introduction}

The syndrome of pyelo-ureteral junction (SJPU) or hydronephrosis is the most frequent malformations of the urinary tract system. The incidence of congenital 
hydronephrosis is about 5 per 100,000 births [1].

SJPU corresponds to a dilation of the pyelocalicielles cavities upstream of an obstacle situated at the level of the pyelo-ureteral junction [2]. The etiology is often congenital. It is either an aperistaltic junction segment, or aberrant vessels or a high insertion ureter [1] [3].

The standard treatment for SJPU remains open pyeloplasty according to the Anderson-Hynes technique [4]. This technique has a success rate greater than $90 \%$ [1] [3].

Nowadays, the management of SJPU is marked by the development of so-called mini-invasive techniques which are laparoscopic pyeloplasty and endopyelotomies.

In Congo, an earlier study on the diagnosis and treatment of pyelo-ureteral junction syndrome in 13 cases was published in 2004 by Bouya, et al. [5]. Our work proposed to study the Anderson-Hynestechnic [4] in order to evaluate the results obtained and the short, medium and long term complications.

\section{Patients and Methods}

\subsection{Framework and Methods of Study}

This was a retrospective study on the medical records of patients operated for an anomaly of the pyeloureteral junction, in the urology-andrology department of University Hospital, Brazzaville, from 2000 to 2014. Diagnostic of pyelo-ureteral junction syndrome was based on ultrasonography, urography and tomography result.

We included in the study any patient with pyelo-ureteral junction syndrome, operated by Anderson-Hynes [4] open pyeloplasty, who had a complete medical record. Operated patients whose records were incomplete were not included. We classified hydronephrosis into 4 types as proposed by CendrenMoulard and Valeyer. Type1: localized dilatation with pelvis; Type 2: dilation of the pelvis and calices, but rapid impregnation of the cavities and good thickness of the parenchyma; Type 3: Large pelvic-calyx dilation with a fuzzy and incomplete image within normal delays, very clear thinning of the cortex; Type 4: silent kidney [6]. In total, 36 files were the subject of this study.

The variables studied were: whether preoperative nephrostomy was performed, the approach used for pyeloplasty, intraoperative exploration, associated procedures, intraoperative drainage (transnephro-anastomotic or transpyelo-anastomotic drain), intraoperative incidents, duration of interventions, average duration of drains and bladder catheter, average length of hospital stay, short-term complications, medium and long-term complications, follow-up (clinical intravenous urography and tomograhy) after average of 12 months.

After the discharge of the service, the patients were reviewed at 1 month for a clinical control. During this check, the presence of residual pain and the condition of the wound was assessed. At three months, clinical and ultrasound control was performed. At 6 months, antomograhy was performed to assess the permeability of the anastomosis and ureter. Clinical and ultrasound control was per- 
formed at 1 year and every year up to 3 years. The result of the surgery was considered a "success" when there was both the disappearance of clinical signs and the permeability of the ureteropulmonary anastomosis to intravenous urography, that is to say the passage of the contrast product throughout the ureter in normal time and without hydronephrosis.

\subsection{Characteristics of the Population}

During the study period, 36 patients met our inclusion criteria.

The average age of our patients was 29.4 years, extreme ( 7 and 53 years). There was male predominance in $63.9 \%$ (23 cases) with a $2 / 1$ sex ratio. The patients came from Brazzaville in the majority of cases (32 cases) Pointe-Noire (3 cases) and Kinkala (1 case).

Clinically, the circumstances of discovery were multiple. Flank or lumbar pain was the most common form of discovery (28 cases). The other circumstances were repeated urinary tract infection (5 cases), hematuria (2 cases) and abdominal mass (1 case).

Physical examination was normal in 29 cases. He had noted a large kidney in 1 case; a sensitivity of the lumbar fossa in 6 cases.

Paraclinically, cytobacteriological examination of urine (ECBU) was performed in all patients. It had allowed isolating a germ in 6 cases. It was an Escherichia coli (4 cases) and Klebsiella pneumoniae (2 cases). These urinary tract infections had been treated by antibiotic therapy in relation to the results of the antibiogram. This examination is performed systematically in all our patients preoperatively to sterilize the urine before any intervention.

\section{Results}

Preoperative nephrostomy was performed in 11 patients. The surgical approach was the lombotomy in the 11th intercostal space in all cases.

Intraoperative exploration revealed pyelo-ureteral junction stenosis in all cases, peripelitis in two patients (5.5\%) with pyeliclithiasis. In three patients $(8.3 \%)$, there was evidence of an inferior polar vessel crossing the pyelo-ureteral junction.

Resection of the pyelo-ureteric junction, sometimes associated with remodeling of the renal pelvis, followed by pyelo-ureteral anastomosis using the Anderson Hynes technique. The pyelo-ureteral anastomosis was made by two suture contin. The suture material used was vicryl ${ }^{\circledR}$ Absorbable Yarn 4/0.

Two patients (5.5\%) underwent pyelolithotomy for pyeliclithiasis associated with the treatment of pyelo-ureteral junction syndrome.

Three patients $(8.3 \%)$ had an aberrant lower polar vessel decay (Figure 1 ) associated with treatment.

The drainage was transpyeloanastomotic in $56 \%$ of the cases, transurethral in $17 \%$ of the cases, and in $28 \%$ of the cases, it did not have drainage. The drainage of the renal lodge by a Redon drain was systematic in all cases (36 cases).

Peroperatively, we noted as incident: a case of peritoneal breccia (2.8\%) and a case $(2.8 \%)$ of pleural breccia. 


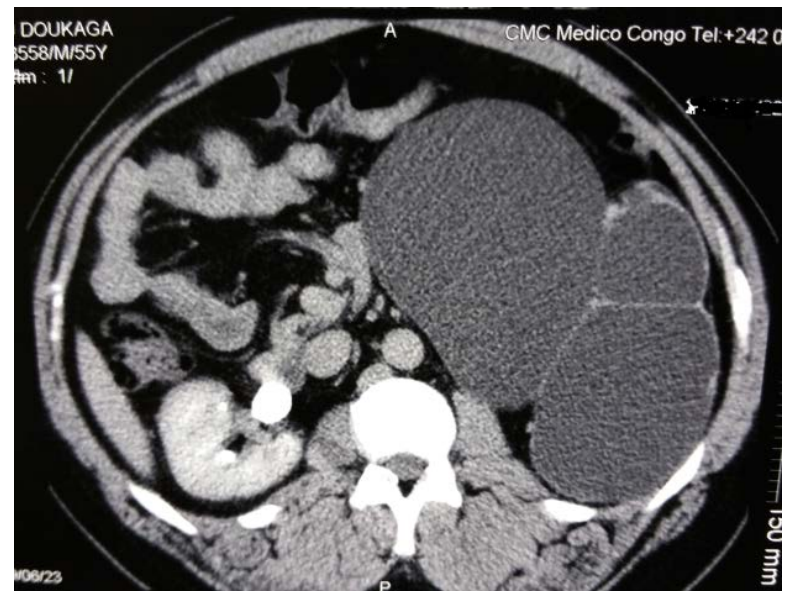

Figure 1. Left giant hydronephrosis in a 45 -year-old patient.

The average duration of interventions was $119 \pm 15 \mathrm{~min}$, extreme (94 - 237 min). The postoperative monitoring parameters are shown in Table 1.

Patients were followed for an average of 12 months with extremes of 6 and 24 months.

The 35 patients (97.2\%) had an improvement in symptomatology with disappearance of lumbar or flank pain and other functional signs. One patient (2.8\%) had persistent low back pain.

The results of intravenous urography at 6 months of postoperative follow-up are shown in Table 2.

In the short term, seven patients had complications. It was a parietal suppuration in 3 cases, treated by antibiotic adapted to the isolated germ, and a urinary leakage by the operative wound in 4 cases supported by drainage of the urine.

In the medium and long term, one patient (2.8\%) had pyelo-ureteral stenosis. The success rate of pyeloplasty was $97.2 \%(\mathrm{n}=35)$.

Renal function was explored in all patients by the determination of blood creatinine. No cases of renal failure had been revealed. The radiological assessment makes it possible to establish the diagnosis of hydroneplorosis. Ultrasound was performed in all patients. It revealed a dilatation of the renal cavities in all cases. In addition, she had diagnosed 2 cases of pyelic calculus.

Intravenous urography was performed in all patients. It allowed making the diagnosis in all cases. It is the same as for urography performed in 7 cases (Figure 2). The attack was unilateral in 34 cases; right in 21 cases and left in 13 cases. Urography was performed in 13 patients in our study. He confirmed the diagnosis in all these cases (Figure 2). Two cases of pyelic computation had been diagnosed by urography, three cases of inferior polar aberrations had been found. The syndrome of the pyelo-ureteral junction was bilateral in 2 cases. Only the symptomatic side was operated. Taking into account the classification of CENDREN MOULARD and VALAYER, pyelo-ureteral junction syndrome was type 1 ( 0 cases); type 2 in 14 cases (38.9\%), type 3 in 21 cases (58.3\%) and type 4 in 1 case $(2.8 \%)$. 


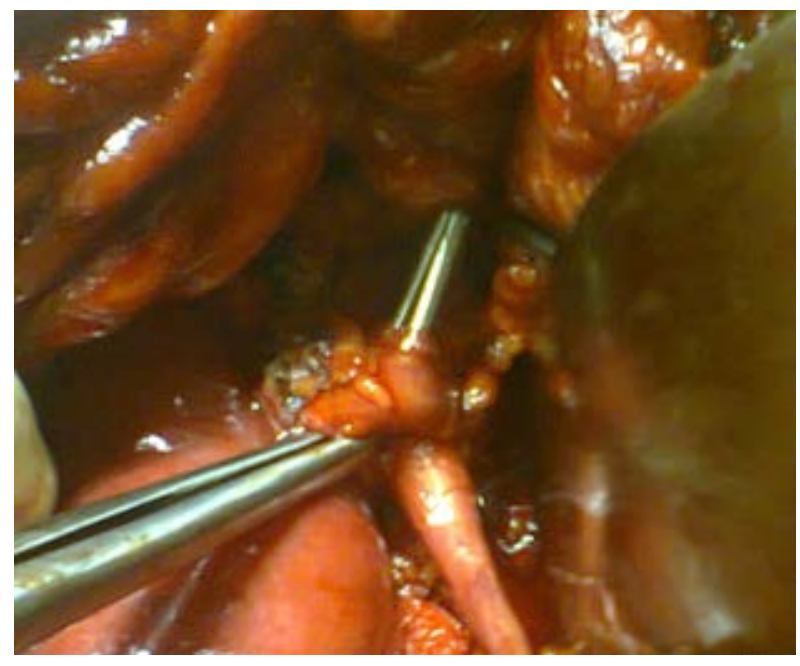

Figure 2. Intraoperative image of a polar vessel crossing the pyelo-ureteral junction.

Table 1. Result postoperative monitoring parameters.

\begin{tabular}{ccc}
\hline Parameters & Average duration & Extreme \\
\hline Duration hospitalization & 11.2 days & $7-30$ days \\
Transanastomotiquedrain & 11.3 days & $5-24$ days \\
Drain of renal lodge & 12.8 days & $6-25$ days \\
Bladder survey & 2.2 days & $1-3$ days \\
\hline
\end{tabular}

Table 2. Results of intravenous urography at 6 months according to the grade of hydronephrosis.

\begin{tabular}{ccccc}
\hline \multicolumn{5}{c}{ Results of IVU at 6 months } \\
\hline $\begin{array}{c}\text { Grade of } \\
\text { l'hydronéphrosis }\end{array}$ & $\begin{array}{c}\text { Number of } \\
\text { patients }\end{array}$ & $\begin{array}{c}\text { Secretion } \\
\text { within } \\
\text { normal time }\end{array}$ & $\begin{array}{c}\text { Decrease in } \\
\text { pyelocalical } \\
\text { dilatation }\end{array}$ & $\begin{array}{c}\text { Pyeloureteral } \\
\text { passage }\end{array}$ \\
\hline I & 0 & 0 & 0 & 0 \\
II & 14 & 14 & 14 & 14 \\
III & 21 & 21 & 20 & 1 \\
IV & 1 & 1 & 1 & 20 \\
\hline
\end{tabular}

\section{Discussion}

Open pyeloplasty according to the Anderson-Hynes technique associates the resection of the stenosedpyelo-ureteric junction with a pyelo-ureteral anastomosis. The open surgical approach was the standard treatment for SJPU since1949, its description by Anderson-Hynes [4]. This technic had been improved by René Kuss. It makes to treat SJPU as well as associated pathologies (removal of pyeliclithiasis, uncrossing of the inferior polar pedicle) [1] [3].

Other techniques exist, Fenger's plasty, Foley's Y-V plasty which have the distinction of not removing the non-functional area, but repair the stenotic portion by increasing functional area with pyelic's flap. 
Lombotomy was the first route used in all our patients. Extraperitoneal and extrapleural is the most commonly used approach in open pyeloplasty [1] [3] [5]. Diao et al. [7] performed lombotomy in $83.4 \%$ of cases. It has the advantage of combining a good exposure on the pyelo-ureteral junction, to allow an excellent section of the pelvis and to realize an impervious anastomosis [3]. However, it has the disadvantage of causing non-esthetic scars, causing significant postoperative pain and causing parietal weakness and evenenation [2] [8] [9]. To avoid these complications of lombotomy, other minimally invasive surgical techniques have been developed (laparoscopy, endopyelotomy) giving functional results comparable to open surgery [10] [11] [12].

In our study, two patients (5.56\%) underwent pyelotithomy with pyeloplasty for pyelic computation. Bentani et al. [13] found a renal calculus associated with SJPU in $17.8 \%$ of cases.

We have considered the pyelo-ureteral junction syndrome as the factor favoring the occurrence of pyelic calculus. However, several authors believe that the stasis of urine alone is not enough to explain the occurrence of lithiasis: the urinary metabolic factors (hypercalciuria) of lithogenesis are necessarily associated [14]. In addition, in three patients (8.3\%), there was evidence of an inferior polar vessel crossing the pyelo-ureteral junction. These patients had undergone a decrease in the inferior polar vessel associated with pyeloplasty. These results are comparable to those observed by Kirakoya et al. [15], who found rates of $11.4 \%$. These vessels have been demonstrated in $30 \%$ to $70 \%$ of cases of pyelouretral junction syndrome [16]. The implementation of transpyelo-ureteral drainage associated with drainage of the renal lodge is recommended [3], but there is no consensus regarding drainage. Excretory drainage seems to be an immediate safety factor, limiting the risk of fistula and urinoma. It does not affect the long-term prognosis and the occurrence of recurrence. In our study, one case of peritoneal breccia and 1 case $(2.78 \%)$ of pleural breccia were observed. The peritoneal gap had been treated peroperatively by suture. The pleural breccia had been repaired immediately. The frontal chest $\mathrm{x}$-ray performed on day 1 postoperative had not revealed a pneumothorax. These incidents are also reported in the literature in similar proportions [7]. The haemorrhagic incidents have been described [16].

The mean duration of the intervention in our study was $119 \pm 15 \mathrm{~min}$. This operating time is comparable to those reported in the literature (90 - $120 \mathrm{~min})$ [1] [3] [7]. The operative time in open surgery is generally lower than that of laparoscopic pyeloplasty. This operating time in laparoscopic pyeloplasty varies from 180 to $480 \mathrm{~min}$ in the literature [1]. The mastery of laparoscopic techniques by the different teams has improved the operating time. Thus Bentani et al. in Morocco [13] find an average operative time of $175.5 \mathrm{~min}$. Soulie et al. [18], in France, an average time of $150 \mathrm{~min}$. The average hospital stay in our study was 11.2 days with extreme 7 - 30 days. This stay is comparable to those reported in the literature: between 10 to 12 days [1] [2] [14] [16].

However, the average stay was significantly reduced by laparoscopic surgery 
between 3 and 5 days [1] [13] [17] [18]. This laparoscopic pyeloplasty allows a rapid recovery of activity. In our study, seven patients had short-term complications. Diao et al. [7] had 20\% short-term complications, including 3 cases (10\%) parietal suppuration and 3 cases of urohematoma (10\%). Short-term complications of pyeloplasty are usually urinary leakage in the anastomosis, urinary fistulas and urinomas [1] [3]. These complications are found both during the PCO and laparoscopically [19].

Open pyeloplasty gives very good results (disappearance of clinical signs, normalization of urographic images) with a long-term success rate of $90 \%$ to $100 \%$ [1] [3] [5]. In our series, a case of failure (pyelo-ureteral stenosis) was noted. This stenosis is due to periuretal fibrosis. Percutaneous nephrostomy was performed while waiting for pyeloplasty to be resumed. The failures most often correspond to persistent stenosis, either by insufficient resection, periuretal fibrosis, non-sloping anastomosis or neglect of a lower polar pedicle, and sometimes an error of indication (retention kidney very deficient) [3].

The results of PCO are currently comparable to those of laparoscopic pyeloplasty [13] [18] [20].

\section{Conclusion}

Anderson-Hynes open pyeloplasty remains the treatment of choice for SJPU in our context with excellent results. But its indications tend to decrease in favor of laparoscopic pyeloplasty, which reproduces the Anderson-Hynes technique with comparable functional results and less morbidity. Thus, the arrival of laparoscopic and endoscopic surgery in our therapeutic arsenal is necessary because it will contribute to the reduction of the length of hospitalization of patients.

\section{Conflicts of Interest}

The authors declare no conflicts of interest regarding the publication of this paper.

\section{Ethical Clearance}

This paper obtained the ethical clearance of the ethical comity of health sciences number 00000077/MRS/DGRST/CERSSA.

\section{References}

[1] Cormier, L., Lefèvre, F., Gaucher, O. and Mourey, E. Mangin P. (1999) Anomaly of the Pyelo-Ureteraljunction and Hydronéplorosis. Elsevier, Paris.

[2] Ferhi, K., Rouprêt, M., Rode, J., Misraï, V., Lebeau, T., Richard, F. and Vaessen, C. (2009) Technical Aspects of Laparoscopic Robot-Assisted Pyeloplasty. Progrès en Urologie, 19, 606-610.

[3] Carpentier, X. and Amiel, J. (2008) Adult Pyelo-Uretral Junction Syndrome: Open Surgical Treatment. EMC Elsevier, Masson SAS, Paris.

[4] Anderson, J.C. and Hynes, W. (1949) Retrocavalureter: A Case Diagnosed Peroperatively and Treated Successfully by Plastic Opération. British Journal of Urology, 
21, 209-211. https://doi.org/10.1111/j.1464-410X.1949.tb10773.x

[5] Bouya, P.A. and Makosso, E. (2004) Diagnosis and Treatment of Pyelo-Ureteral Junction Syndrome: About 13 Cases. Journal Africain d Imagerie Médicale, 7, 485-488.

[6] Valayer, J., Cendron, J. and Petit, P. (1967) Congenital Pyelo-Caliceal Due to Anomaly of the Pyelo-Ureteral Junction in Children. Surgical Treatment. Annales de Chirurgie Infantile, 8, 99-117.

[7] Diao, B., Fall, B., Kaboré, F.A., Sow, Y., Sarr, A., Thiam, A., Fall, P.A., Ndoye, A.K., Bâ, M. and Diagne, B.A. (2012) Anderson-Hynes Open Pyeloplasty: Which Indications in the Area of Laparoscopic Surgery? Progrès en Urologie, 22, 1010-1014. https://doi.org/10.1016/j.purol.2012.08.274

[8] Zhang, X., Li, H.Z., Ma, X., Zheng, T., Lang, B., et al. (2006) Retropective Comparison of Retroperitoneal Laparoscopic versus Open Dismembered Pyeloplasty for Ureteropelvic Junction Obstruction. Journal of Urology, 2, 388-392.

[9] O’Reilly, P.H., Brooman, P.J., Mak, D., Jones, M., Pickup, C., Atkinson, C. and Pollard, A.J. (2001) The Long-Term Results of Anderson-Hynes Pyeloplasty. BJU International, 87, 287-289. https://doi.org/10.1046/j.1464-410x.2001.00108.x

[10] Shalhav, A.L., Mikhail, A.A., Orvieto, M.A., Gofrit, O.N., Gerber, G.S. and Zorn, K.C. (2010) Adult Stentless Laparoscopic Pyeloplasty. Journal of the Society of Laparoendoscopic Surgeons, 11, 8-13.

[11] Robert, E., Aubry, E., Pecoux, F., Priso, R.H., Sfeir, R. and Besson, R. (2010) Pyeloplasty for Pyelo-Ureteric Junction Syndrome in Children: Lombo-Assisted Procedure versus Lombotomy. Progrès en Urologie, 20, 219-223.

https://doi.org/10.1016/j.purol.2009.08.036

[12] Wang, P., Xia, D., Ma, Q. and Wang, S. (2014) Retroperitonéal Lapaoscopic Management of Uroteropelvic Junction in Patients with Horseshoe Kidney. Urology, 84, 1351-1354. https://doi.org/10.1016/j.urology.2014.07.029

[13] Bentani, N., Moudouni, S.M., Wakrim, B., Amine, M., Hanich, T., et al. (2012) Laparoscopic Repair of Pelviureteric Junction Obstruction: Results and Keys to Success during the Learning Curve. African Journal of Urology, 18, 49-54. https://doi.org/10.1016/j.afju.2012.04.011

[14] Saussine, C., Lechevalier, E. and Traxer, O. (2008) Urolithiasis and Ureteropelvic Junction Obstruction-Urétérale. Progrès en Urologie, 18, 986-988. https://doi.org/10.1016/j.purol.2008.09.002

[15] Kirakoya, B., Kabore, F.A., Zango, B., Pare, A.K., Yameogo, C. and Kambou, T. (2015) Management of Ureteropelvic Junction at the Urology Department of University Hospital Yalgado Ouedraogo (Burkina Faso). Uro' Andro, 1, 148-152.

[16] Stern, J.M., Park, S., Anderson, J.K., Landman, J., Pearle, M. and Cadeddu, J.A. (2007) Functional Assessment of Crossing Vessels as Etiology of Ureteropelvic Junction Obstruction. Urology, 69, 1022-1024. https://doi.org/10.1016/j.urology.2007.02.055

[17] Martin, X., Gelet, A., Cuzin, B., Badet, L. and Colombel, M. (2010) Ureteropelvic Junction Obstruction. Contribution of Robotic Asssted Syrgery. E-mémoires de l'académie Nationale de Chirurgie, 9, 70-73.

[18] Soulie, M., Seguim, P., Cartron, G., Mouly, P., Vazzoler, N. and Plante, P. (2001) La pyéloplastie par Retroperitoneoscopic Pyeloplasty for Primary Hydronephrosis: Preliminary Results of the First 30 Procedures. Progrès en Urologie, 11, 625-630.

[19] Wakabayashi, Y., Johnin, K., Kataoka, A., Kil, C.J., Yoshiki, T. and Okada, Y. (2002) Initial Failure in Open Pyeloplasty for Ureteroplelvic Junction Obstruction. Hinyo- 
kika kiyo. Acta urologica Japonica, 48, 457-475.

[20] Gogus, C., Karamursel, T., Tokatli, Z., Yoman, O., Ozdiler, E. and Gögüuss, O. (2004) Long Term Results of Anderson-Hynes Pyéloplasty in 180 Adults in the Era of Endourologic Procedures. Urologia Internationalis, 73, 11-14.

https://doi.org/10.1159/000078796 